\title{
Long-term survival of adrenal metastasis from non-small cell lung cancer
}

\author{
Akihiko Iwase $\cdot$ Emi Onuma $\cdot$ Osamu Nagashima \\ Toshifumi Yae $\cdot$ Makiko Kunogi $\cdot$ Shu Hirai
}

Received: 28 February 2012/ Accepted: 23 July 2012/Published online: 28 August 2012

(C) The Japan Society of Clinical Oncology 2012

\begin{abstract}
Long-term survival of patients who undergo surgical resection of isolated adrenal metastasis from nonsmall cell lung cancer (NSCLC) has been reported. In these patients, primary lung cancers were generally resected or resectable. We report a 50-year-old man of NSCLC (T3N2M1) with locally advanced lung adenocarcinoma and solitary ipsilateral adrenal metastasis, which was detected by FDG/PET (fluorodeoxyglucose/positron emission tomography). After the thoracic lesions had been controlled with chemo-radiation therapy for 2 years, the remaining adrenal metastasis was surgically resected. He was disease-free for 7 years. Our case suggests that solitary adrenal metastasis may be resectable in NSCLC patients controlled by chemo-radiation therapy, and FDG/PET is useful to detect and follow adrenal metastasis.
\end{abstract}

Keywords Non-small cell lung cancer · Solitary adrenal metastasis - Surgical resection - Fluorodeoxyglucose/ emission tomography

\section{Introduction}

Adrenal metastases from non-small cell lung cancer (NSCLC) are usually found in patients with other sites of

\footnotetext{
A. Iwase $(\bowtie) \cdot$ E. Onuma $\cdot$ O. Nagashima · T. Yae .

M. Kunogi

Division of Respiratory Medicine, Juntnedo Tokyo Koto

Geriatric Medical Center, Shin-suna 3-3-20, Koto-ku, Tokyo

136-0075, Japan

e-mail: iwaseaki@juntendo.gmc.ac.jp

S. Hirai

Division of Pathology, Juntendo Tokyo Koto Geriatric Medical

Center, Shin-suna 3-3-20, Koto-ku, Tokyo 136-0075, Japan
}

distant metastasis. However, several small studies have reported that an adrenalectomy for isolated adrenal metastasis in NSCLC, with a surgical resection of the primary lung cancer, can achieve long-term survival [1]. We described long-term survival after resection of solitary adrenal metastasis in a patient with NSCLC whose thoracic lesions were controlled with chemo-radiation therapy.

A 50-year-old man was referred to our hospital due to chest X-ray shadow. He complained of knock pain on the left back. At admission his chest X-ray showed a bulky mass in the apex of the left lung (Fig. 1). Chest CT revealed that the tumor invaded the left subclavicular artery and the chest wall with mediastinal lymphadenopathy (Fig. 2). Brushing specimen obtained from the tumor by bronchoscopy tested positive for adenocarcinoma. Fluorodeoxyglucose/positron emission tomography (FDG/PET) showed abnormal uptake in the left adrenal gland and the primary site (Fig. 3). He was diagnosed as having a primary lung cancer (T3N2M1), with solitary adrenal metastasis. Radiation therapy was performed on the thorax and the left adrenal gland with chemotherapy comprising carboplatin and paclitaxel. After the chemo-radiation therapy, the thoracic lesions were induced to complete remission, but the left adrenal metastasis remained. Although we changed the chemotherapy combination with carboplatin from paclitaxel to vinorelbine and next gemcitabine and continued for 2 years, the adrenal lesion was enlarged in the follow-up FDG/PET (Fig. 4). In this period, relapse of the primary lesions and other metastasis were not recognized. Therefore left adrenalectomy was performed. The resected tumor was poorly differentiated adenocarcinoma with central necrosis. In immunohistochemical analysis, the tumor cells tested negative for thyroid transcription factor-1 (TTF-1) and napsin A, and epidermal growth factor receptor (EGFR) mutation was not recognized. However, the adrenal tumor had a clear margin with normal 


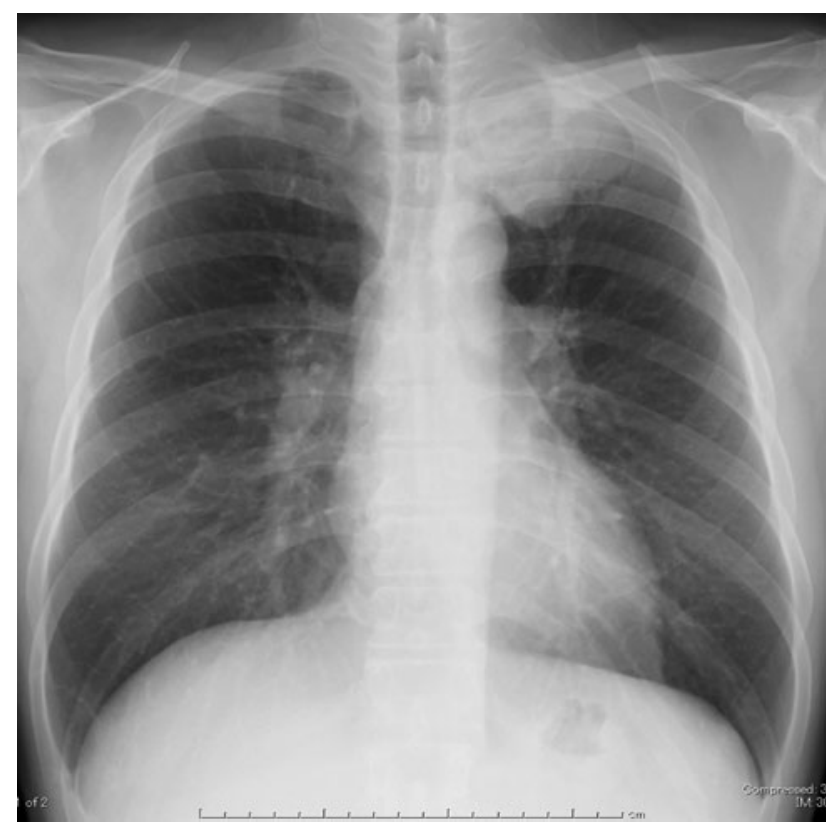

Fig. 1 Chest X-ray at admission revealed a bulky mass in the apex of the left lung

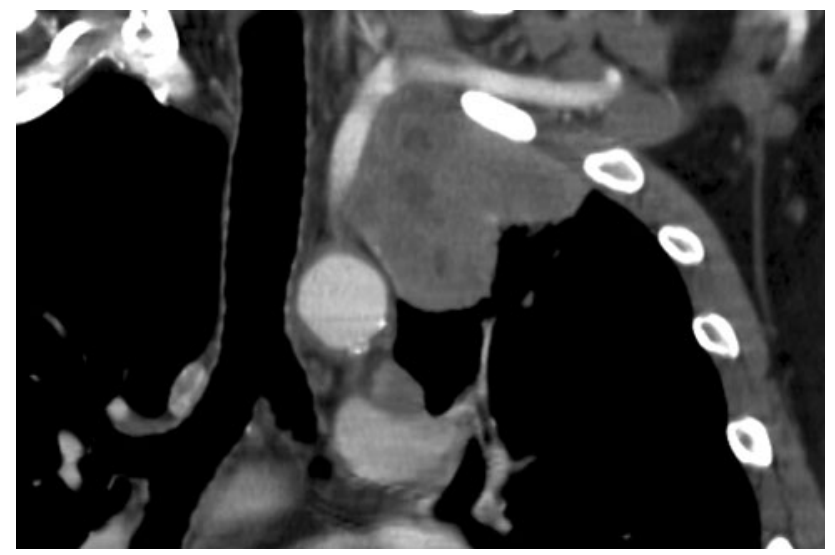

Fig. 2 Chest CT revealed that the tumor invaded the left subclavicular artery and the chest wall with mediastinal lymphadenopathy

adrenal tissue, which was compatible with metastatic adenocarcinoma (Fig. 5). No other tumor emerged during the observation period. We then considered the adrenal tumor to be metastasis from the lung adenocarcinoma. After the operation he was treated with oral TS-1 for 2 years. He has survived disease-free for 7 years from the initial visit and 5 years after the operation.

\section{Discussion}

In case reports and limited retrospective studies, adrenalectomy was recommended for an isolated adrenal metastasis

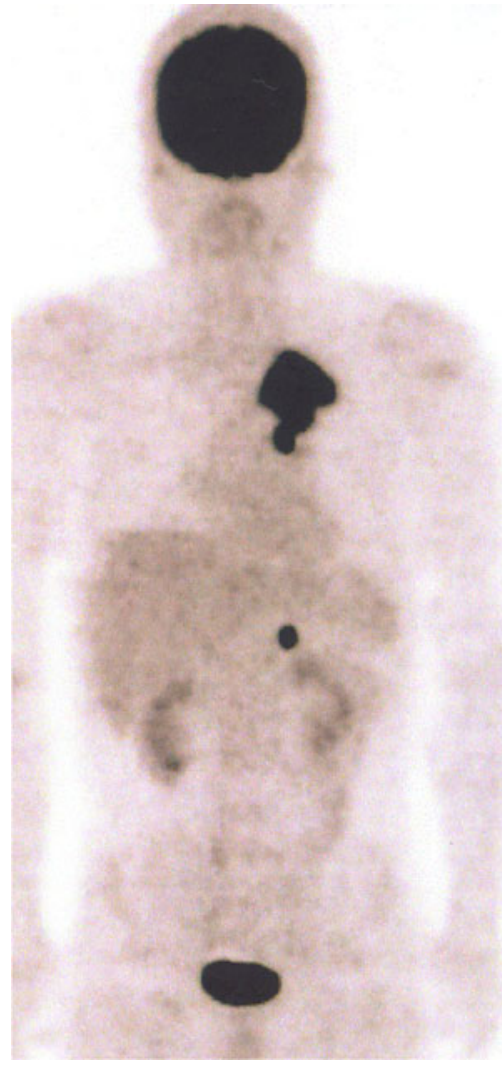

Fig. 3 FDG/PET showed abnormal uptake in the left adrenal gland and the primary site

from NSCLC, when a primary lesion was resected or resectable [1-4]. NCCN Guideline version 2.2012 announced that solitary adrenal metastasis from NSCLC is resectable when the primary site is in T1-2, N0-1 or T3, N0 [5]. In our case, the lung tumor invaded the thoracic wall and left subclavicular artery. Mediastinal lymph node involvement was also recognized. Thereupon, chemo-radiation therapy on both thoracic lesion and adrenal metastasis was selected. Although the thoracic lesions were well controlled in our patient for 2 years, the adrenal metastasis has remained. Therefore, we performed adrenalectomy, allowing him to achieve disease-free, long-term survival. Our investigation showed that adrenalectomy should be considered as a therapeutic option for patients whose primary lung tumor is not only operated on, but also controlled by chemoradiation therapy in the long term. Porte et al. [2] initially reported that $\mathrm{N} 2$ involvement (ipsilateral mediastinal lymphadenopathy) was a poor prognosis predictor, but they could not find clinical and pathological criteria in their later study [3]. In a recent report, Raz et al. [4] suggested that no patients with contralateral adrenal metastasis and mediastinal nodal disease survived long-term after adrenalectomy. They also showed that the time interval between the primary lung cancer and adrenal metastasis was not significantly associated with survival. Tanvetyanon et al. [6] compared 


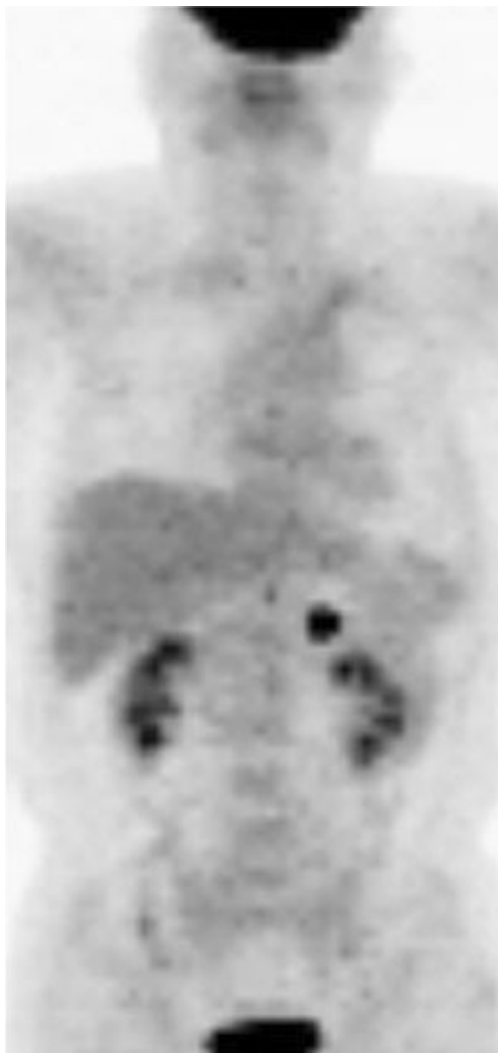

Fig. 4 Although the thoracic lesions were induced to complete remission, the adrenal lesion was enlarged in the follow-up FDG/PET

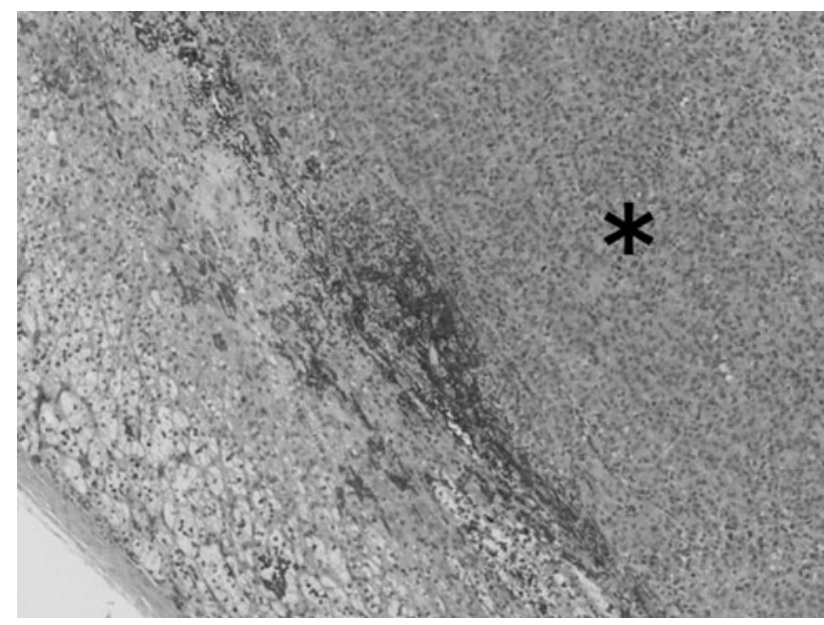

Fig. 5 Resected tumor (asterisk) was poorly differentiated adenocarcinoma, which had a clear margin with normal adrenal tissue

isolated synchronous with metachronous adrenal metastasis in a systemic review. They showed that for isolated adrenal metastasis, patients with a synchronous metastasis from NSCLC who underwent adrenalectomy had a shorter median survival than those with a metachronous metastasis. However, the 5-year survival was achieved in approximately
$25 \%$ in both groups. Karolyi [7] postulated that in the early stages adrenal metastases from lung cancer developed mainly by lymphogenous, but later mainly by hematogenous routes with investigation of autopsied lung cancer cases. This study supports the notion that surgical resection of solitary adrenal metastasis from NSCLC provides a survival benefit in well-selected patients.

Recently, FDG/PET has been available for general surveillance and management of malignant tumors. Some investigators reported that FDG/PET is an accurate, noninvasive technique for differentiating benign from metastatic adrenal lesions detected on $\mathrm{CT}$ in patients with lung cancer [8]. We predict that solitary adrenal metastasis will be detected more easily and earlier in many patients with NSCLC in the future. Although our patient's cancer was stage T3N2, long-term control of the primary lesions was obtained under chemo-radiation. In general, locally advanced NSCLC was treated with concurrent chemo-radiation therapy. Median survival time and 5-year survival were about 20 months and less than $20 \%$, respectively [9]. There are no selection criteria to identify the subgroup who will benefit from surgical resection of solitary adrenal metastasis from NSCLC controlled by chemo-radiation. Further controlled prospective studies are necessary to resolve the problem.

Conflict of interest No author has any conflict of interest.

\section{References}

1. Pfannschmidt J, Dienemann H (2010) Surgical treatment of oligometastatic non-small cell lung cancer. Lung cancer 69:251-258

2. Porte HL, Roumilhac D, Graziana JP et al (1998) Adrenalectomy for a solitary adrenal metastasis from lung cancer. Ann Thorac Surg 65:331-335

3. Porte H, Siat J, Guibert B et al (2001) Resection of adrenal metastases from non-small cell lung cancer: a multicenter study. Ann Thorac Surg 71:981-985

4. Raz DJ, Lanuti M, Gaissert HC et al (2011) Outcomes of patients with isolated adrenal metastasis from non-small cell lung carcinoma. Ann Thorac Surg 92:1788-1793

5. NCCN (2012) NCCN Guidelines: non-small cell lung cancer version 2.2012. Available via NCCN. www.nccn.com. Accessed $26 \mathrm{Feb} 2012$

6. Tanvetyanon T, Robinson LA, Schell MJ et al (2008) Outcomes of adrenalectomy for isolated synchronous versus metachronous adrenal metastases in non-small-cell lung cancer: a systemic review and pooled analysis. J Clin Oncol 26:1142-1147

7. Karolyi P (1990) Do adrenal metastases from lung cancer developed by lymphogenous of hematogenous route? J Surg Oncol 43:154-156

8. Kumar R, Xiu Y, Yu JQ et al (2004) ${ }^{18}$ F-FDG PET in evaluation of adrenal lesions in patients with lung cancer. J Nucl Med 45:2058-2062

9. Yamamoto N, Nakagawa K, Nishimura Y et al (2010) Phase III study comparing second- and third-generation regimens with concurrent thoracic radiotherapy in patient with unresectable stage III non-small-cell lung cancer: West Japan Thoracic Oncology Group WJTOG 0105. J Clin Oncol 28:3739-3745 